(C) 2014. The Institution of Engineering and Technology. This paper is a postprint of a paper submitted to and accepted for publication in Electronics Letters and is subject to Institution of Engineering and Technology Copyright. The copy of record is available at IET Digital Library

Manuscript for Review

\title{
Electrical conductivity measurement in Thiel-embalmed tissue model: relevance to radiofrequency ablation
}

\begin{tabular}{|r|l|}
\hline Journal: & Electronics Letters \\
\hline Manuscript ID: & Draft \\
\hline Manuscript Type: & Letter \\
\hline Complete List of Authors: & nata \\
& $\begin{array}{r}\text { Wang, Zhigang; University of Dundee, Institute for Medical Science and } \\
\text { Technology } \\
\text { Luo, Hongyan } \\
\text { Nick, Matthias; University of Dundee, } \\
\text { Gueorguieva, Mariana; University of Dundee, } \\
\text { Andre, Pascal; University of St Andrews, } \\
\text { Baker, Richard; University of St Andrews, } \\
\text { McLean, Donald; University of Dundee, } \\
\text { Brown, Stuart; University of Dundee, } \\
\text { Cuschieri, Alfred; University of Dundee, }\end{array}$ \\
\hline Keywords: & $\begin{array}{l}\text { ELECTRICAL CONDUCTIVITY, BIOMEDICAL MEASUREMENT, BIOTHERMICS, } \\
\text { BIOPHYSICS }\end{array}$ \\
\hline & \\
\hline
\end{tabular}




\section{Electrical conductivity measurement in Thiel-embalmed tissue model: relevance to radiofrequency ablation}

Z. Wang, H. Luo, M. Nick, M. Gueorguieva, P. Andre, R.T. Baker, D. McLean, S. Brown and A. Cuschieri

Thiel-embalmed human cadaveric specimens are used widely for biomedical scientific investigation. This letter reports electrical impedance measurements of Thiel-embalming solution from $1 \mathrm{~Hz}$ to 32 $\mathrm{MHz}$. Compared to other solutions studied, Thiel solution has the lowest impedance throughout the test spectrum. The electrical conductivity of Thiel-embalmed liver sample exhibited a relatively flat frequency response from 100 to $500 \mathrm{kHz}$, which is the frequency range used for electro surgery and radiofrequency tumour ablation (RFA). It measured $5 \mathrm{Sm}^{-1}$ compared to $0.22 \mathrm{Sm}^{-1}$ obtained from ex-vivo fresh pig liver. Using finite element modelling and experimental evaluation, ablation zone obtained from the Thiel-embalmed liver sample was extremely small due to its much higher conductivity. Hence, we conclude that Thiel-embalmed tissue cannot be used as a reliable model for RFA evaluation.

Introduction: The standard post-mortem storage of human cadaveric specimens for biomechanical investigation is deep freezing at low temperatures $\left(-20^{\circ} \mathrm{C}\right)$. However, tissue banks of deep frozen human tissues is costly, of limited supply, requires defrosting before usage, and can act as a source of disease transmission during sample handling, unless safety handling precautions are in place. The use of Thiel embalming, recently popularised has overcome these problems as exemplified by the use of Thiel-embalmed heads for assessment of middle ear studies [1].

Thiel embalming first described by Thiel in 1992 [2], conserves texture, elasticity and colour in cadavers close to that of the living and is bactericidal. Unfortunately, Thiel embalming is not widely practised for a variety of reasons, including expertise needed, costs and time required for this soft embalming process. Hence, its availability is confined to a few centres worldwide [3,4]. Dundee University' Thiel-embalming facility, offers Thiel-embalmed cadavers for teaching, surgical skill training and research and development of medical devices [5]. There have been conflicting reports regarding the influence of Thiel preservation on tissue mechanics [6]. Although Thiel' embalmed tissue maintains soft tissue consistency and colour close to that of living tissue, Fessel et al. [7] reported that Thiel embalmed tendons do not exhibit the same biomechanical characteristics of fresh frozen tendons (from partial denaturation by boric acid in Thiel solution). There is little known in the reported literature on the electrical/dielectric properties of Thiel embalmed tissue. This report addresses this issue by providing data on electrical conductivity measurement. It also discusses the unsuitability of Thiel embalmed human tissue for studies and R \& D in radiofrequency ablation (RFA).

Thiel embalming solution and impedance measurement: Table 1 lists composition of our Thiel (tank) fluid, together with one moisturiser for impedance measurement comparison.

Table 1: Composition of the Thiel tank solution.

\begin{tabular}{|c|c|c|}
\hline & Moist & Tank fluid \\
\hline Hot tap water & $20 \mathrm{~L}$ & $20 \mathrm{~L}$ \\
\hline Boric acid & $600 \mathrm{~g}$ & $720 \mathrm{~g}$ \\
\hline Ammonium nitrate & - & $2.4 \mathrm{~kg}$ \\
\hline Potassium nitrate & - & $1.2 \mathrm{~kg}$ \\
\hline Sodium sulphite & $1000 \mathrm{~g}$ & $1.68 \mathrm{~kg}$ \\
\hline Propylene glycol & $1 \mathrm{~L}$ & $2.4 \mathrm{~L}$ \\
\hline Stock II* & $200 \mathrm{~mL}$ & - \\
\hline Formalin $(8.9 \%)$ & - & $2.04 \mathrm{~L}$ \\
\hline
\end{tabular}

* Stock II: $1 \mathrm{~kg}$ Chlorocresol in 10 litres of glycol.
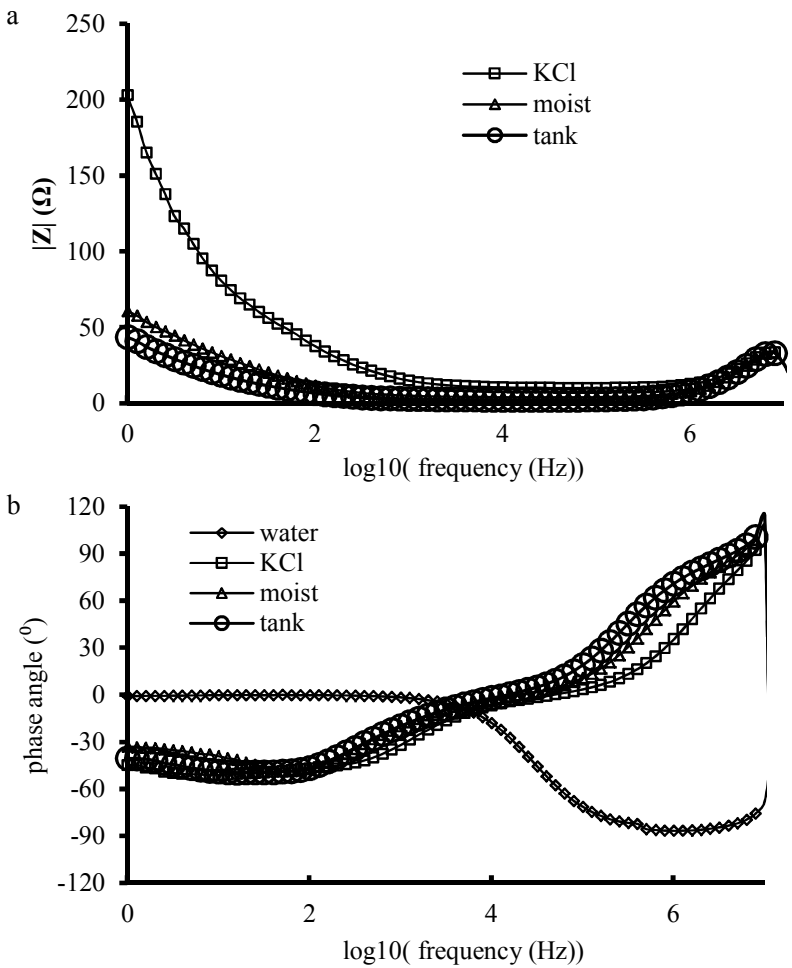

Fig. 1 Impedance of selected solutions: (a) magnitude and (b) phase angle in the measurement frequency range.

Distilled water and aqueous $\mathrm{KCl}$ solution $(11.5 \mathrm{~g} / \mathrm{L})$ were used as control samples. A sample cell consisted of two electrodes with 314.2 $\mathrm{mm}^{2}$ cross-section area and separated by a distance of $10 \mathrm{~mm}$, yielding a sample fluid volume of $3142 \mathrm{~mm}^{3}$ (or $3.142 \mathrm{~mL}$ ) [8]. A Solartron 1260 Frequency Response Analyser was used to obtain impedance spectra of samples over a frequency range from $1 \mathrm{~Hz}$ to $32 \mathrm{MHz}$ (Fig. 1). Impedance magnitude of the distilled water was extremely high (> $120 \mathrm{k} \Omega$ ) at low frequency up to $10 \mathrm{kHz}$, and was not included in Fig. 1a. Compared to all tested samples, Thiel solution (tank) has the lowest impedance magnitude throughout the whole test spectrum. For example at $10 \mathrm{kHz}$, impedances were $2.3 \Omega, 4.0 \Omega, 10.0 \Omega$ and $120.0 \mathrm{k} \Omega$ from tank, moist, $\mathrm{KCl}$ and water samples, respectively (Fig. 1a).

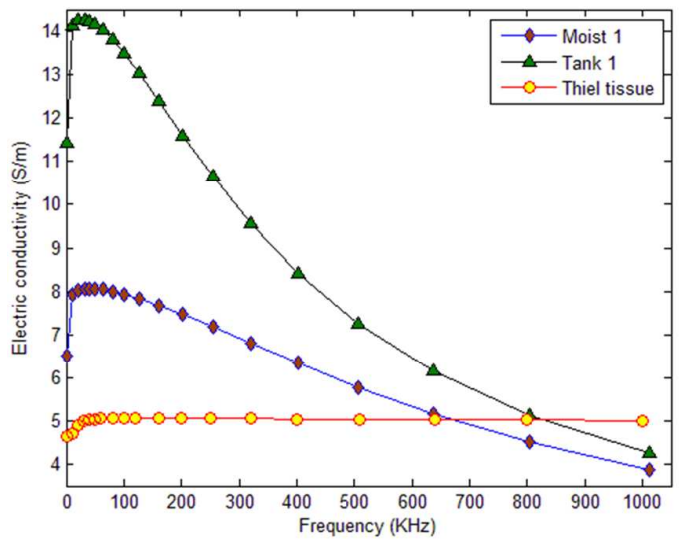

Fig. 2 Electrical conductivity of Thiel-embalmed human cadaveric liver from 1 to $1000 \mathrm{kHz}$, plotted together with the two solutions in Table 1 .

Thiel embalmed cadaveric tissue conductivity in terms of suitability for use in radiofrequency ablation (RFA) studies: RFA has been widely used in minimally invasive ablative cancer therapy, particularly for liver cancer [9]. Hence Thiel embalmed liver samples were used in the study. A custom cylinder sample holder was designed with two removable (by screw action) electrodes $\left(\mathrm{A}=113 \mathrm{~mm}^{2}\right.$ cross-section area) separated by a distance $\mathrm{L}$ of $35 \mathrm{~mm}$, yielding a sample fluid volume of $3955 \mathrm{~mm}^{3}$. Several sample holders were constructed and separately used for 
different types of tissue samples (e.g., human cadaver, ex-vivo porcine tissue or tissue-mimicking gel-phantom). An Agilent Impedance Analyzer (model 4395A), which has high frequency range up to 500 $\mathrm{MHz}$, was used for tissue conductivity measurements. Since this study was specifically concerned with RFA application which normally uses frequencies only up to $500 \mathrm{kHz}$, we selected frequency points from 1 $\mathrm{kHz}$ to $1 \mathrm{MHz}$ for the measurement of tissue electrical conductivity. Electrical conductivity of the measured liver sample (explanted Thielembalmed cadaveric liver, IRIS L-Liver_FEB-2011) had a relatively flat frequency response (Fig. 2) and was around $5 \mathrm{Sm}^{-1}$ from $100 \mathrm{kHz}$ to $500 \mathrm{kHz}$ (most used frequency range for electro-surgery and RFA). Although it was much lower than conductivities measured from Thielsolutions (see Tank1 and Moist1, Fig. 2), Thiel-embalmed liver tissue still had 10 times higher electrical conductivity than that obtained from ex-vivo fresh pig liver (Table 2). Our measured conductivity of around $0.22 \mathrm{Sm}^{-1}$ for ex-vivo fresh pig liver aligns with values from literature, i.e., $0.23 \mathrm{Sm}^{-1}[10]$.

Table 2: Comparison of liver conductivity measurements at $500 \mathrm{kHz}$, average and standard deviation were deduced from $\mathrm{n}=10$ measurements.

\begin{tabular}{|c|c|}
\hline Thiel-embalmed cadaveric liver & Ex-vivo fresh pig liver \\
\hline $5.10 \pm 0.56 \mathrm{Sm}^{-1}$ & $0.22 \pm 0.08 \mathrm{Sm}^{-1}$ \\
\hline
\end{tabular}

RFA in FEM modelling and experimental evaluation:

RFA probe (RITA Model 30) was used for both finite element method (FEM) analysis using COMSOL Multiphysics package and experimental evaluation together with an electrosurgical generator (DRE ASG-300) [11]. Ex-vivo pig liver samples were used for comparison (Fig. 3). We also measured thermal conductivities of Thielembalmed and fresh pig liver samples and found that there was no significant difference between them $(p=0.05)$. Our measured value of $0.501 \mathrm{Wm}^{-1} \mathrm{~K}^{-1}(\mathrm{n}=10, \mathrm{SD}=0.08)$ was used in our FEM modelling which is in agreement with [12]. Details of our thermal conductivity data will be published elsewhere.
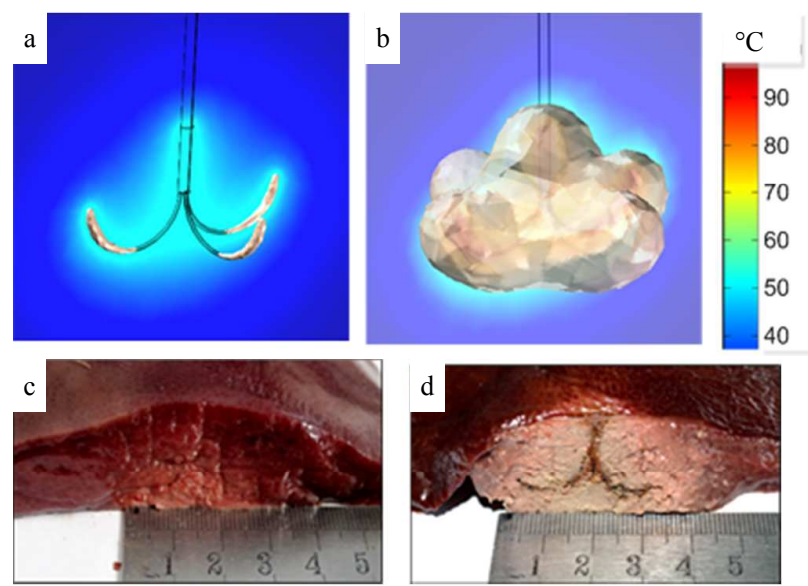

Fig. 3 RFA using ex-vivo pig liver model with $(a, c)$ Thiel-embalmed and $(b, d)$ fresh: FEM simulation and experiment evaluation.

Using ex-vivo fresh liver model, at an applied RF energy of $6 \mathrm{~kJ}$ (i.e., $20 \mathrm{~W}$ and $300 \mathrm{~s}$ ), the maximal temperature at the ablation zone was kept just below $100{ }^{\circ} \mathrm{C}$ with the ablation volume shown as in iso-thermal surface plot of over $50^{\circ} \mathrm{C}$ (Fig. $3 \mathrm{~b}$ ) and tissue color changes $(>3 \mathrm{~cm}$, Fig. 3d). However, due to much higher electrical conductivity for the Thiel-embalmed tissue, there was no obvious ablation zone in both FEM (Fig. 3a) and experiment (Fig. 3c), even with longer ablation time (900 s). For the Thiel-embalmed tissue, higher conductivity means lower resistivity resulting in lower resistive heating in RFA. Heating patterns would be more diffuse and tend to deliver less heating to the target zone. Finally, the RF generator outputs less power when the load ( $<5 \Omega$, the Thiel-embalmed liver) is too low compared to the nominated output with load $(>150 \Omega)$.
Conclusion: Electrical conductivities of Thiel-embalming solutions and embalmed liver samples were measured. On the basis of the present study, because of much higher electrical conductivities of Thielembalming solutions and embalmed tissues, we conclude that Thielembalmed tissue is not a suitable model for RFA studies and R \& D.

Acknowledgments: This article presents independent research partially commissioned by the National Institute for Health Research (NIHR) under Invention for Innovation (i4i) Programme. PA gratefully acknowledges SUPA for funding his Advanced Research Fellowship. The views expressed in this article are those of the authors and not necessarily those of the NHS, the NIHR or the Department of Health.

Z. Wang, H. Luo, M. Nick, M. Gueorguieva, D. McLean, S. Brown and A. Cuschieri (Institute for Medical Science and Technology, University of Dundee, Dundee DD2 1FD, UK)

E-mail: z.z.wang@dundee.ac.uk

P. Andre, R. T. Baker (University of St Andrews, St Andrews, UK)

H Luo: also with Chongqing University, Chongqing, China.

\section{References}

1. Stieger, C., Candreia, C., Kompis, M., et al.: 'Laser Doppler vibrometric assessment of middle ear motion in Thiel-embalmed heads', Otol. Neurotol., 2012, 33, (3), pp. 311-318, doi: 10.1097/Mao.0b013e3182487de0

2. Thiel, W.: 'The preservation of the whole corpse with natural color', Ann. Anat., 1992, 174, (3), pp. 185-195

3. Benkhadra, M., Gerard, J., Genelot, D., et al.: 'Is Thiel's embalming method widely known? A world survey about its use', Surg. Radiol. Anat., 2011, 33, (4), pp. 359-363, doi: 10.1007/s00276-010-0705-6

4. Wolff, K., Kesting, M., Mucke, T., et al.: 'Thiel embalming technique: a valuable method for microvascular exercise and teaching of flap raising', Microsurgery, 2008, 28, (4), pp. 273-278, doi: 10.1002/micr.20484.

5. Prasad Rai, B., Tang, B., Eisma, R., et al.: 'A qualitative assessment of human cadavers embalmed by Thiel's method used in laparoscopic training for renal resection', Anat. Sci. Educ., 2012, 5, (3), pp. 182-186, doi: $10.1002 /$ ase 1267

6. Benkhadra, M., Faust, A., Ladoire, S., et al.: 'Comparison of fresh and Thiel's embalmed cadavers according to the suitability for ultrasound-guided regional anesthesia of the cervical region', Surg. Radiol. Anat., 2009, 31, (7), pp. 531-535, doi: 10.1007/s00276-0090477-z

7. Fessel, G., Frey, K., Schweizer, A., et al.: 'Suitability of Thiel embalmed tendons for biomechanical investigation', Ann. Anat., 2011, 193, (3), pp. 237-241, doi: 10.1016/j.aanat.2011.03.007

8. Chen, S., Maclaren, D., Baker, R., et al.: 'Inhomogeneous Composition of Alloyed Iron-Platinum Magnetic Nanoparticles Synthesized at Low Temperature', J. Mat. Chem. 2011, 21, pp. 36463654, doi: 10.1039/c0jm03165h

9. Chu, K.F., Dupuy, D.E.: 'Thermal ablation of tumours: biological mechanisms and advances in therapy', Nat. Rev. Cancer, 2014, 14, (3), pp. $199-208$, doi: $10.1038 / \mathrm{Nrc} 3672$

10. Gabriel, C., Peyman, A., Grant, E.H.: 'Electrical conductivity of tissue at frequencies below $1 \mathrm{MHz}$ ', Phys. Med. Biol., 2009, 54, (16), pp.4863-4878, doi: 10.1088/0031-9155/54/16/002

11. Wang, Z., Aarya, I., Gueorguieva, M., et al.: 'Image-based 3D modeling and validation of radiofrequency interstitial tumor ablation using a tissue-mimicking breast phantom', Int. J. Comput. Assist. Radiol. Surg., 2012, 7, (6), pp. 941-948. doi: 10.1007/s11548-0120769-3

12. Tungjitkusolmun, S., Staelin, S.T., Haemmerich, D., et al.: 'Threedimensional finite-element analyses for radio-frequency hepatic tumor ablation', IEEE Trans. Biomed. Eng., 2002, 49, (1), pp. 3-9, doi: $10.1109 / 10.972834$ 